\title{
Darwinian Philosophy as Optimization Method for Design High Reflection Mirror Include New Merit Function
}

\author{
Alaa N.Abudalghaffar*
}

Date of acceptance 28/2/2010

\begin{abstract}
:
In this paper we proposes the philosophy of the Darwinian selection as synthesis method called Genetic algorithm ( GA ), and include new merit function with simple form then its uses in other works for designing one of the kinds of multilayer optical filters called high reflection mirror. Here we intend to investigate solutions for many practical problems. This work appears designed high reflection mirror that have good performance with reduction the number of layers, which can enable one to controlling the errors effect of the thickness layers on the final product, where in this work we can yield such a solution in a very shorter time by controlling the length of the chromosome and optimal genetic operators

Result shows that the construction of multilayer high reflection mirror using in this approach can be considered as a master stone for design another type of filters with most complicated performance, and it is difficult designing in other approach The experiment results demonstrate that our approach is a powerful technique. It is enable to locate the global optimum optimal automatically with high confidence without need for a good starting design.
\end{abstract}

Key words: Multilayer optical filter, genetic algorithm, synthesis high reflection mirror, optimisation multilayer coating

\section{Introduction}

High reflection mirror is one of the important multilayer optical filters(MOFs) (MOFs)are coating which consist of stack of thin layers of materials with difference in refractive indices[1].Depending on the total number of layers composition and thickness of each layer.

The multilayer optical coating has numerous applications for example in scientific instrument manufacturing, spectroscopes medicine and astronomy $[1,2]$.

High reflection mirror are used to change the spectral intensity distribution or the state of polarization of the electromagnetic radiation incident for satisfying performance specifications. There are many different optical filter design approaches, which are often required for the solutions of different types of problem. These approaches can be roughly divided into analytical, graphical, and numerical methods. [3] Numerical methods are now the most widely used design techniques. Refinement methods [4] and synthesis methods[3,4] are two approaches of numerical optical coating .Refinement methods need a starting design which should be close to the optimal design, otherwise no good results are obtained. Synthesis methods are more general, they create a promising design without a starting

*Physics Department, College of Science for Women, University of Baghdad. 
design. This promising design can be refined afterwards, a recent development for designing MOFs is the introduction of genetic algorithms (GA s) [5 ] .In this paper we use genetic algorithms, to optimize high reflection mirror. Genetic algorithms (GAs) [6,7] are optimization technique used in evolutionary computing, inspired by Darwin's theory of evolution [7]; it uses a model similar to the natural process of biological to produce good specialized genotypes through the selection, crossover and mutation operators.(GAs) $[7,8]$ can be classified as a synthesis method and do not require a starting design ,they have been applied to fit interference multilayer reflectivity for optimizing the design of high reflection mirror filters $[7,8,9]$.

Almost all numerical methods for the design of optical multilayer are based on the using of merit function. In this work, we used the a new type of merit function and this is the first using in this kind of optimization methods and has very simple mathematic form, this will be expressed in the section (3.1.2). The present work introduces several improvements for designing high reflection mirror with GAs, Solutions found by GAs are improved by refinement method based on Hooke \& Jeaves search, by this technique we could design these mirror which guide us to design more complex filters, in just few layers of coating with flexible and faster. In this method, it's important to know the genetic operators, Darwinian philosophy, and the maximum number of layers.

\section{Statement of the problem}

The calculation of the interference effects of a system of thin film can be accomplished simply by considering a ray approach in which beams are reflected backwards and forwards between the various interfaces. In the steady state these rays combine to form the resultant reflected or transmitted ray. This is very straightforward to implement numerically. The technique usually employed [ 10 ] involves a two _ stage process in which the electromagnetic wave in each film are reduced to two partial waves, one positive and negative going these partial waves transfer the total electric and magnetic of the rear interface of each layer to the forward interface. This transfer reduces to a matrix multiplication, and the calculation of the multiplayer properties reduces to a series of matrix operations .

We consider a series of thin films, each having an index of refraction $n_{j}$ and a thickness $\mathrm{z}_{\mathrm{j}}$. Incident light is incident normally for an absorption - free medium (usually air) and strikes the top ( layer M ) of an M-layer film the last (layer 1) is adjacent to the substrate ( see figure (1)) the matrix expression from which the total reflectance is 
computed, is $\mathrm{y}_{\mathrm{J}}$, and $\mathrm{z}_{\mathrm{J}}$ are the optical admittance refractive index, and physical thickness, respectively of the jth thin film .In addition $y_{\text {sub }}$ is the optical admittance of the substrate. As long as there is on absorption in the medium, the admittance and refractive indices can be taken to be the same .

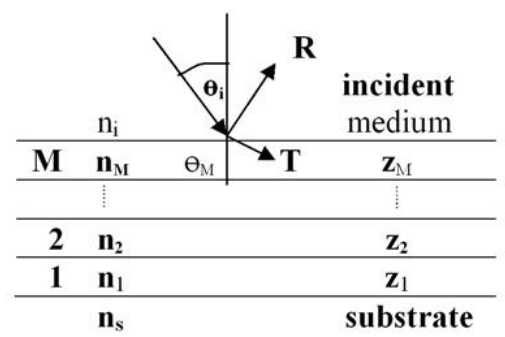

Figure 1.An optical multilayer system

The value of $B$ and $C$ represent the total electric and magnetic field amplitudes a particular frequency. In terms of them, the total reflectance of the multiplayer structure is found by $\mathrm{R}$

$\mathrm{R}=\left|\frac{\mathrm{Y}_{0} \mathrm{~B}-\mathrm{C}}{\mathrm{Y}_{0} \mathrm{~B}+\mathrm{C}}\right|^{2}$

With Yo representing the admittance of the incident medium.

\section{Theory}

\section{Genetic Algorithms}

GAs are a special class of global optimizers, based on the theory of evolution. A GA is able to minimizes (or maximize) a function $\mathrm{F}(\mathrm{x})$, where(x) represents a parameter vector, by searching the parameter space of $\mathrm{x}$ for the optimal solution $[7,8,9]$.GAs do not operate on single trial solution, but on a group of solution called a population.

A solution, which is called a string, is a vector of all parameters, which are to be optimized. Using evolutionary inspired operators such as fitness, crossover and mutation, the best solutions are modified and passed on to the next generation. In this way the population as a whole moves towards better solutions, ideally to the global optimum. For a better understanding of GAs the reader is referred

to [8] in the fact that individuals having higher performance have greater probabilities to reproduce mimicking acrider parody of Darwinian evolution is the mean point of the algorithm. Other individuals tend slowly to disappear. So one can hope that from two individuals a better one (i.e with a higher performance ) can be made .

\subsubsection{Representation}

The first step is to general an initial population .In this each member of this population will be a binary string of length $\mathrm{L}$ that corresponds to the problem encoding. Then the crossover and mutation can be applied of each string refereed to as genotype or alternatively, chromosome and the initial population is generated randomly. A trial solution, containing values of all parameters that are to be optimized, can be represented by a vector of bits where integer values. A design consisting of $\mathrm{M}$ layers is completely described by a vector, containing the thicknesses of each layer $\mathrm{X}=\mathrm{X} 1, \ldots \ldots, \mathrm{X}_{\mathrm{M}}$. There for the search space for designing high reflection mirror consists of integer values for the thickness, in this paper only the length of the chromosome (string) has to be specified, in this way there is no thickness zero, then the number of layers in a design is flexible, and the GA can decide how many layers actually will be present in a design, when applying the crossover operator during a GA run, it is now 
possible to copy complete blocks from one string to another.

\section{Merit function}

Merit function, is single value express the current performance of the system and direction the flow of future calculations. So, the optimize design command tells the program to vary the thickness of the layers or their position so that the merit function is minimized. In this papers merit function is design to determined by calculating the corresponding reflections spectrum using the matrix formalism [11].This spectrum is compared with a target reflection spectrum by using function $\mathrm{F}$, which sums the difference between the intensities of the reflection spectrum, indicated by $\mathrm{R}_{\mathrm{i}}$, and the target spectrum, indicated by $\mathrm{R}_{\mathrm{T}}$ as shown in eq( 3.1$)$.

$$
\mathrm{F}=\left|\mathbf{R}_{\mathbf{i}}-\mathbf{R}_{\mathrm{T}}\right| \leq \gamma
$$

Where $\gamma \approx 1 * 10^{-4}$ or $5 * 10^{-3}$

The resulting merit function $\mathrm{F}$ is minimized by the GA. By changing the target reflection spectrum $R_{T}($ $\lambda$ ).It is possible to construct filters with specific properties.

optical thickness and the best achievable average reflection [ 12,], i.e. the quality of the design. In this paper we are interested in the reflection $\mathbf{R}$ of high reflection optical multilayer filter.

The general problem in the design is to find the design parameters (thicknesses to variable (known) refractive indices). We have applied the GA to design high reflection mirror with just only four layers, as shown in figures ( $2 \mathrm{a}-\mathrm{e}$ ). Also these figures show the optima

\subsection{Refinement with Hook \&} Jeeves (H \& J ) method.

GAs are able to locate promising regions for global optima in a search space, but sometime have difficulty finding the exact minimum of these optima [ 10]. Especially, when the search space for constructing reflecting filter is very large, like that design , can improved by a second optimization method .In this paper, a procedure based on H\&J method, is used to refine the designs. According to Dobrowolski [11 ], a refinement is defined in the following way: "for a given problem, it is possible to reach a satisfactory solution by gradually modifying its construction parameters . Such a process is called refinement"

A large number of such refinement methods are compared by Dobrowolski and kamp (1990) [ 11 ]. On three different problems, they were not able to identify a single best method, but recommended the choice of the Hooke_ Jeeves or damped least squares method.

\section{Experimental Results}

GA are applied to the synthesis of multilayer optical filter (MOFs), GA is not a tailored heuristic , i.e.. need no optical knowledge or a special starting design ,the optimization results confirm theoretical consideration that predict a relationship between the thickness of the layer which can be essentially to affect the good solution convergence of the optimization spectral reflectance of this coating systems, it is specified in table ( 1 ). These figures shown that high reflection mirror can be consider as a base design to other optical filter types like edge filters.

Figures (3a-c) presented (offer) the optimization physical thickness of design reflectance curve of the high 
reflection mirror with only 7 layers specified in the table ( 2 ). When we point a length of the string (chromosome) we pass the most problems difficulties arise in the design the (MOfs) when the number of layer of coating become large .Figures $(3 a-c)$ refers to the cold and hot mirrors, and gave in few layers reflection approach $90 \%$, this is able to obtain acceptable solutions to design band pass filter .

All figures shown in (2a-e) and (3a-c) designed without need the refinement method .

The fig (4) show the target requirements for design special optical filter and the ability of ( GA-H\&J ) approach for design MOF with nine layer only, with the specific parameters construction and properties of materials for design this filter representation in table ( 3 ). In this case the optimal search of physical thickness based on oblique deposition of the coating .

Table ( 1 ) Parameter of GA and physical properties used in design high reflection mirror coating with four

\begin{tabular}{|c|c|c|c|c|}
\hline $\begin{array}{c}\text { Genetic } \\
\text { properti } \\
\text { es }\end{array}$ & $\begin{array}{c}\text { Length of } \\
\text { chromoso } \\
\text { me } \\
\text { (L) } \\
\mathrm{L}=28 \text { bit }\end{array}$ & $\begin{array}{c}\text { Populati } \\
\text { on size } \\
\text { P. }=20\end{array}$ & $\begin{array}{c}\text { Crossove } \\
\text { r } \\
\text { probabili } \\
\text { ty } \\
n c=0.9\end{array}$ & $\begin{array}{c}\text { Mutatio } \\
\text { n rate } \\
\mathrm{nm}=0.0 \\
1\end{array}$ \\
\hline $\begin{array}{l}\text { Physical } \\
\text { properti } \\
\text { es }\end{array}$ & $\begin{array}{c}\text { Incident } \\
\text { medium } \\
n_{0}=1\end{array}$ & $\begin{array}{l}\text { Substrate } \\
\text { index } \mathrm{n}_{8} \\
=1.51\end{array}$ & \multicolumn{2}{|c|}{$\begin{array}{c}\text { High Refractive } \\
\text { index of layer( } \mathrm{ZnS}) \\
\mathrm{n}_{\mathrm{H}}=2.35 \\
\text { Low Refractive } \\
\text { index of } \\
\text { Layer }\left(\mathrm{MgF}_{2}\right) \mathrm{n}_{\mathrm{L}}= \\
1.38 \\
\text { Number of layers }=4\end{array}$} \\
\hline
\end{tabular}

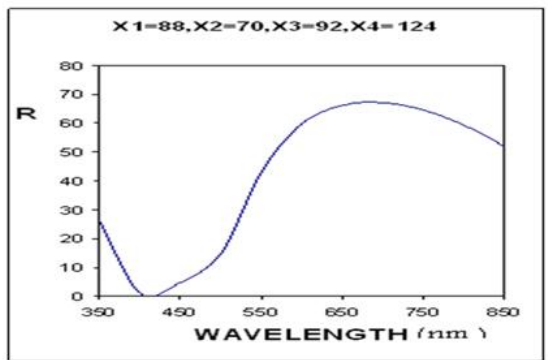

Figures (2-a ) Spectral reflectance profile of four layers solution generated by the GA for an high reflection mirror where $\mathrm{X} 1=88 \mathrm{~nm}, \mathrm{X} 2=70 \mathrm{~nm}$, $\mathrm{X} 3=92 \mathrm{~nm}$.

$\mathrm{X} 4=124 \mathrm{~nm}$. This presents the physical thickness of layers of layers successively

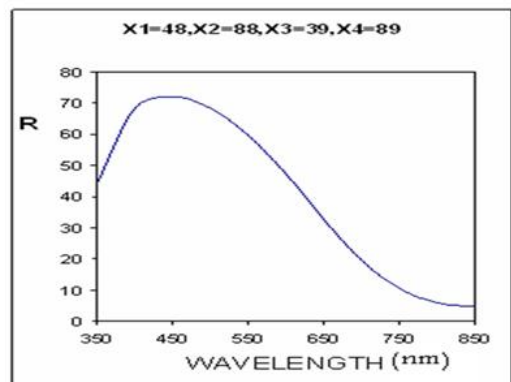

Figures (2-b) Spectral reflectance profile of four layers solution generated by the GA for high reflection mirror where $\mathrm{X} 1=48 \mathrm{~nm}, \mathrm{X} 2=88 \mathrm{~nm}$, $\mathrm{X} 3=39 \mathrm{~nm}$,

$\mathrm{X} 4=89 \mathrm{~nm}$. This presents the physical thickness of layers successively

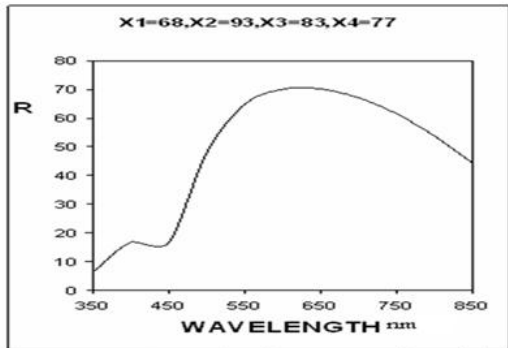

Figures (2-c) Spectral reflectance profile of four layers solution generated by the GA for an high reflection mirror where $X 1=68 \mathrm{~nm}, X 2=93 \mathrm{~nm}$, $\mathrm{X} 3=83 \mathrm{~nm}, \mathrm{X} 4=77 \mathrm{~nm}$. This presents the physical thickness of lavers successively 


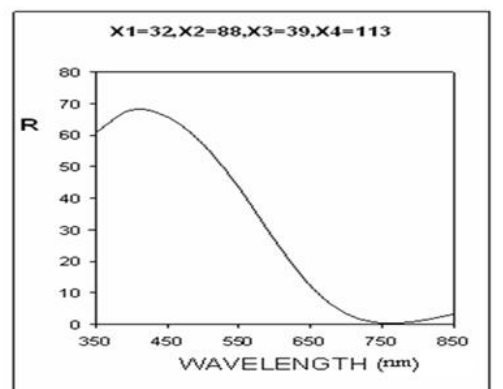

Figures (2-d) Spectral reflectance profile of four lavers solution generated by the GA for an high reflection mirror where $\mathrm{X} 1=32 \mathrm{~nm}, \mathrm{X} 2=88 \mathrm{~nm}$, $\mathrm{X} 3=39 \mathrm{~nm}$.

$\mathrm{X} 4=113 \mathrm{~nm}$. Which presents the physical thickness of layers successively

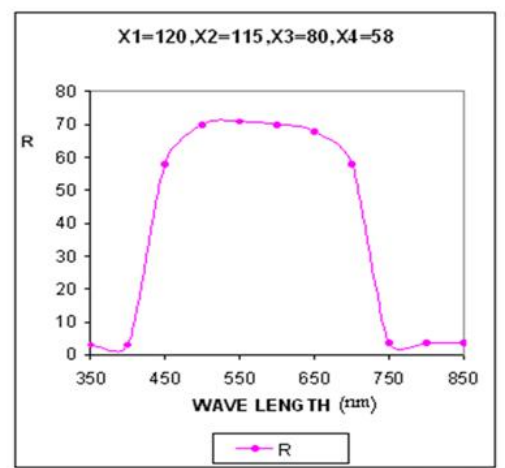

Figures (2-e) Spectral reflectance profile of four layers solution generated by the GA for high reflection mirror where $\mathrm{X} 1=120 \mathrm{~nm}, \mathrm{X} 2=115 \mathrm{~nm}$ , $\mathrm{X} 3=80 \mathrm{~nm}$,

$\mathrm{X} 4=58 \mathrm{~nm}$. This presents the physical thickness of layers successively

Table ( 2) Parameter of GA and physical properties used in design high reflection coating with seven layers.

\begin{tabular}{|c|c|c|c|c|}
\hline $\begin{array}{l}\text { Geneti } \\
\mathrm{c} \\
\text { propert } \\
\text { ies }\end{array}$ & $\begin{array}{c}\text { Length of } \\
\text { chromoso } \\
\text { me } \\
(\mathrm{L}) \\
\mathrm{L}=49 \text { bit }\end{array}$ & $\begin{array}{c}\text { Populat } \\
\text { ion } \\
\text { size. } \\
\text { P.Z }= \\
20\end{array}$ & $\begin{array}{c}\text { Crossov } \\
\text { er } \\
\text { probabil } \\
\text { ity } \\
\text { nc }=0.8\end{array}$ & $\begin{array}{c}\text { Mutati } \\
\text { on rate } \\
\mathrm{nm}=0 . \\
04\end{array}$ \\
\hline $\begin{array}{l}\text { Physic } \\
\text { al } \\
\text { propert } \\
\text { ies }\end{array}$ & $\begin{array}{c}\text { Incident } \\
\text { medium } \\
\mathrm{n}_{0}=1\end{array}$ & $\begin{array}{c}\text { Substrat } \\
\quad \mathrm{e} \\
\text { index } \mathrm{n}_{8} \\
=1.51\end{array}$ & \multicolumn{2}{|c|}{$\begin{array}{l}\text { High Refractive } \\
\text { index of layer( } \\
\mathrm{ZnS}) \mathrm{n}_{\mathrm{H}}=2.35 \\
\text { Low Refractive } \\
\text { index of } \\
\left.\text { Layer( } \mathrm{MgF}_{2}\right) \mathrm{n}_{\mathrm{L}} \\
=1.38 \\
\text { Number of layers } \\
=7\end{array}$} \\
\hline
\end{tabular}

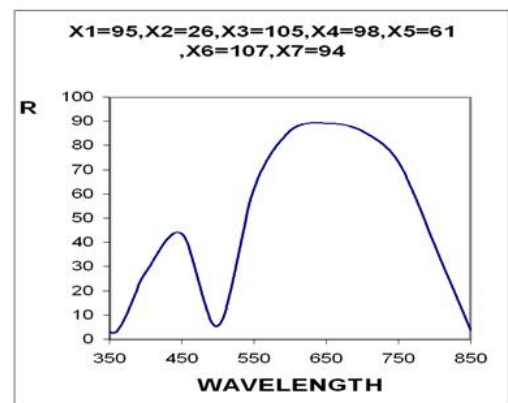

Fig(3-a)Spectral reflectance profile of seven layers solution generated by the GA for high reflection mirror and thickness for each laver with reflective indices $\mathrm{n}_{\mathrm{H}}=2.35, \mathrm{n}_{\mathrm{L}}=1.38$

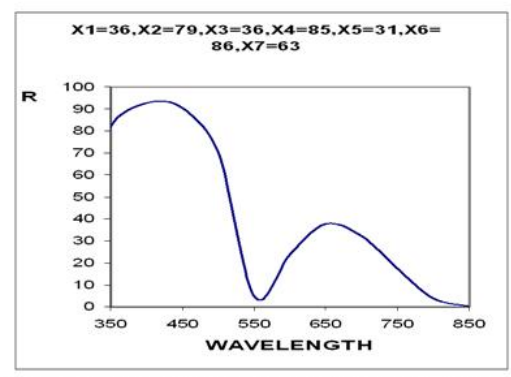

Fig(3-b)Spectral reflectance profile of the seven lavers solution generated by the and GA for high reflection mirror reflective indices $\mathrm{n}_{\mathrm{H}}=2.35, \mathrm{n}_{\mathrm{L}}=$ 1.38

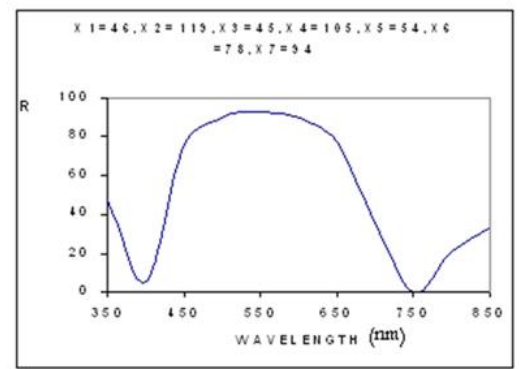

Fig(3-c)Spectral reflectance profile of seven layers solution generated by the seven layers solution generated by the GA for high reflection mirror and thickness for each layer with reflective indices $n_{H}$ $=2.35, \mathrm{n}_{\mathrm{L}}=1.38$ 
Table ( 3 ) Parameter of GA and physical properties used in design MOC with nine layers.

\begin{tabular}{|c|c|c|c|c|}
\hline $\begin{array}{l}\text { Geneti } \\
\mathrm{c} \\
\text { propert } \\
\text { ies }\end{array}$ & $\begin{array}{c}\text { Length of } \\
\text { chromoso } \\
\text { me } \\
\text { ( } \mathrm{L} \text { ) } \\
\mathrm{L}=63 \text { bit }\end{array}$ & $\begin{array}{c}\text { Populat } \\
\text { ion size } \\
P . Z= \\
20\end{array}$ & $\begin{array}{c}\text { Crossov } \\
\text { er } \\
\text { probabil } \\
\text { ity } \\
\text { nc }=0.8\end{array}$ & $\begin{array}{c}\text { Mutati } \\
\text { on rate } \\
\mathrm{nm}=0 . \\
04\end{array}$ \\
\hline $\begin{array}{l}\text { Physic } \\
\text { al } \\
\text { propert } \\
\text { ies }\end{array}$ & $\begin{array}{c}\text { Incident } \\
\text { medium } \\
\mathrm{n}_{0}=1\end{array}$ & $\begin{array}{c}\text { Substrat } \\
\mathrm{e} \\
\text { index } \mathrm{n}_{8} \\
=1.51\end{array}$ & \multicolumn{2}{|c|}{$\begin{array}{c}\text { High Refractive } \\
\text { index of layer( } \\
\mathrm{ZnS}) \mathrm{n}_{\mathrm{H}}=2.35 \\
\text { Low Refractive } \\
\text { index of } \\
\left.\text { Layer( } \mathrm{MgF}_{2}\right) \mathrm{n}_{\mathrm{L}} \\
=1.38 \\
\begin{array}{c}\text { Number of layers } \\
\quad=9\end{array}\end{array}$} \\
\hline
\end{tabular}

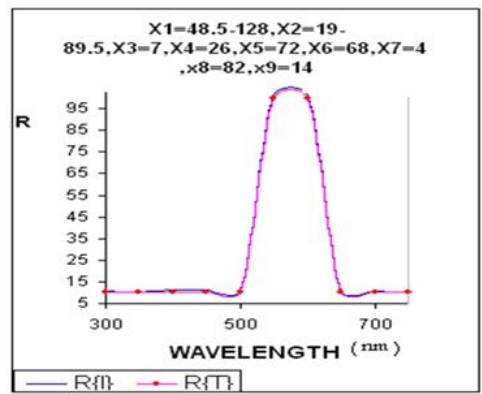

Fig ( 4 ) Spectral reflectance $\mathbf{R}_{\mathbf{i}}$ ( Solid line and the target spectrum $\mathbf{R}_{\mathbf{T}}$ is shown with dotted line profile of nine lavers solution generated by the GA-H \& J procedure for multilayer coating .

Conclusion: In this manuscript the example of genetic algorithms synthesis method for design high reflection mirror, results not always needing a refinement method to improve its solution. Also to design the specification optical filter with a viable, flexible by GA need determined the best length of the string as well as known the optimal genetic operators like the probability of crossover (nc), mutation $(\mathrm{nm})$. In this approach, it is not necessary to specify how many layers will be present in a design, only a maximum layers needs to be defined. Such alternative design method is efficient, fast not construed and this techniqueis still yields solutions of remarkable quality and promises successful applicability to a large number of important industrial problems. so we run the genetic algorithm upon 100 generations, 20 population size. The time required on a medium speed PC was on the order of 1 minute .

\section{References:}

[1] Macleod,H. 2001. Thin -film optical filters.Bristol and Philadelphia: Institute of Physics Publication, $\left(3^{\text {rd }}\right.$ ed.),UAS.P20

[2]Decter,M.2002.The measurements of pollution in the Troposphere (MOPITT)remote sensing instrument, Journal of Atmosphere and Oceanic Technology.19(3): 1772-1776.

[3]Yang,J.M.and Kao,C.Y.2001. Efficient evolutionary algorithm for thin-film synthesis of inhomogeneous optical coatings, J. Applied Optics. 40(19):3256-3267

[4]Dobrowolski, J.A and Kemp, R. A. 1990. Refinement of optical multiplayer system with different optimization procedures,J. Applied Optics.29(3):2876- 2893.

[5] Hagemana, J.A Wehrens, R. Sprang, H.A. and Buydens L.M.C. 2003.

Hybrid genetic algorithm-tabu search approach for optimising multilayer optical coatings, Analytica Chimica Acta 490:211-222

[6]Yang,J.M.and Kao,C.Y. 2000.A robust Evolutionary algorithm for optical thin-film designs, Proceedings of the Congress on Evolutionary Computation, (CEC00). La Jolla, California, USA. p. 978-985

[7] Martin F.S, Frank W.M.and Sameer.C. 2008. Design of multilayer antireflection coatings made from cosputtered and low-refractive-index materials by genetic algorithm, Optical Society of America. 16( 8): 5290 5298. 
[8]Goldberg, D.1989. Genetic Algorithms in Search, Optimization and Machine Learning AdditionWesley, Reading,MA,ed .No.2, pp30 [9] Holland, J. H.1992. Adaptation in Natural and Artificial Systems, MIT Press, MA,ed.No.1,UAS.pp 10

[10] Ables .F. 1950. Recherches sur la propagation des ondes electromagnetiques sinusoidales dans les milieus.Ann.Phys.ser. milieus.Ann.Phys.ser.12(1): 706-784.
[11]Dobrowoliski,J.and Kemp,R.1990.Refinement of optical multilayer systems with different optimization procedures. Applied Optics.29(2):2876-2893

[12] Yang,J.M.and Kao,C.Y. 2000. Integrating adaptive mutations and family competition into genetic algorithms as function optimizer, Soft Computing. 4(2):89-102.

\section{فلسفة دارون كطريقة مثلى لتصميم مرايا الانعكاسية العالية مع أستخدام دالة

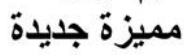

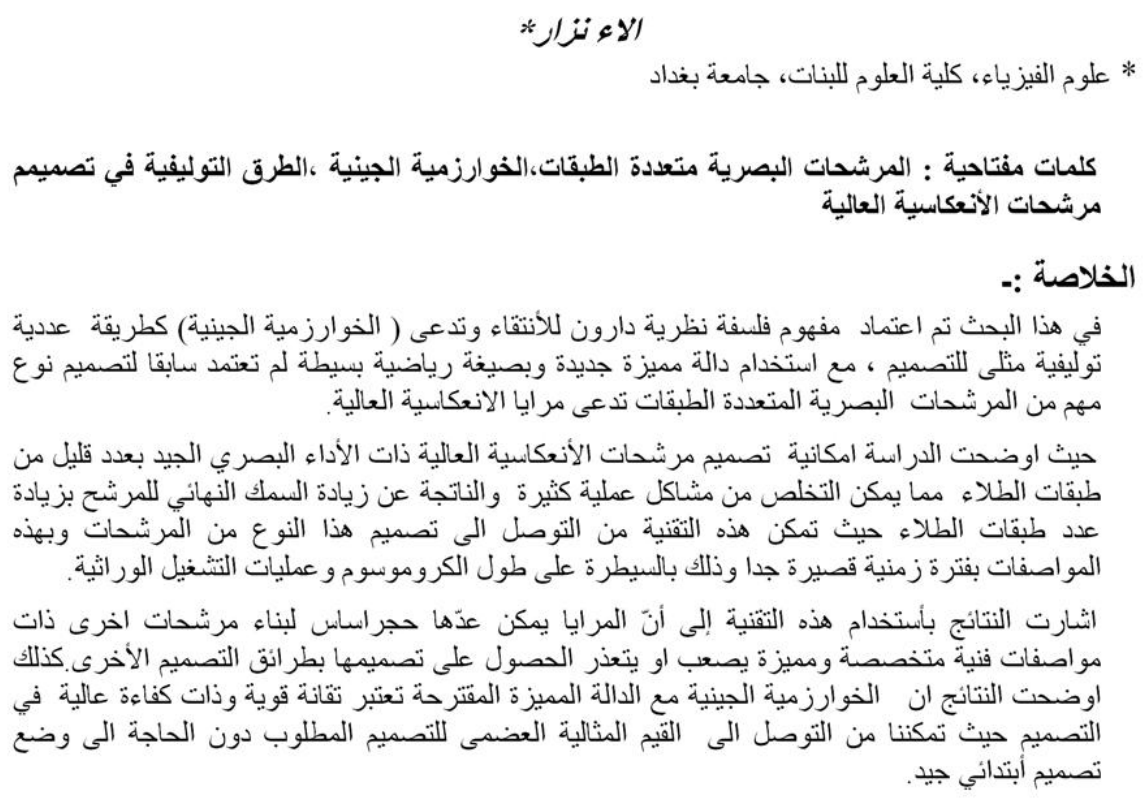

